\title{
Intestinal carriage of vancomycin-resistant Enterococcus spp. among high-risk patients in university hospitals in Serbia: first surveillance report
}

\author{
Ana Janjusevic ${ }^{1 *}\left(\mathbb{D}\right.$, Ljiljana Markovic Denic ${ }^{2}$, Rajna Minic ${ }^{1}$, Anita Grgurevic ${ }^{2}$ and Ivana Cirkovic $^{3}$
}

\begin{abstract}
Background: The screening for intestinal carriage of vancomycin-resistant Enterococcus spp. (VRE) among high risk patients in the Balkan region and molecular epidemiology of VRE is insufficiently investigated, yet it could be of key importance in infection control. The aim of this study was to provide baseline data on VRE intestinal carriage among high-risk patients in Serbian university hospitals, to determine the phenotypic/genotypic profiles of the isolated VRE, to obtain knowledge of local resistance patterns and bridge the gaps in current VRE surveillance.
\end{abstract}

Methods: The VRE reservoir was investigated using stool samples from 268 inpatients. Characterization of isolated VRE stains consisted of BD Phoenix system, genotypic identification, glycopeptide and quinupristin-dalfopristin (Q-D) resistance probing, virulence gene (esp, hyl, efaA, asa 1, gelE, $c p d$ ) detection and MLVA. Biofilm formation was evaluated by the microtiter plate method.

Results: VRE carriage prevalence among at-risk patients was $28.7 \%$. All VRE strains were vanA positive multidrugresistant Enterococcus faecium (VRfm), harboring ermB-1 (38.9\%), esp (84\%), efaA (71.2\%), hyl (54.5\%), asa1 (23.4\%), gelE and $c p d(11.6 \%)$ each. Ability of biofilm production was detected in $20.8 \%$. Genetic relatedness of the isolates revealed 13 clusters, heterogeneous picture and 25 unique MTs profiles.

Conclusion: The obtained prevalence of VRE intestinal carriage among high-risk inpatients in Serbia is higher than the European average, with high percentage of multidrug resistance. The emergence of resistance to Q-D is of particular concern. Close monitoring of pattern of resistance and strict adherence to specific guidelines are urgently needed in Serbia.

Keywords: VRE carriage, MLVA sreening, Antibiotic susceptibility, Serbia

\section{Introduction}

Vancomycin resistant enterococci (VRE) have emerged as one of the most important health-care associated (HA) multidrug-resistant (MDR) pathogens, causing invasive infections, especially in severely ill and

\footnotetext{
*Correspondence: dr.ana.janjusevic@gmail.com

${ }^{1}$ Department of Bacteriology, Institute of Virology, Vaccines and Sera

"Torlak", Vojvode Stepe 458, 11152 Belgrade, Serbia

Full list of author information is available at the end of the article
}

immunocompromised patients [1-3]. Limited therapeutic options for the treatment of VRE infections, the ability of VRE to survive in hospital environment, the capability to colonize the digestive tract of patients and the capacity for acquiring and transferring resistance genes along with the danger of vancomycin-resistant Staphylococcus aureus occurrence are the reasons that VRE was identified as an ESKAPE pathogen (Ente-

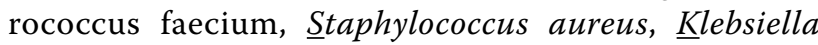

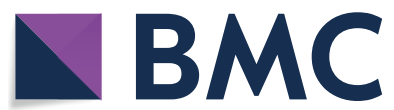

(c) The Author(s) 2021. This article is licensed under a Creative Commons Attribution 4.0 International License, which permits use, sharing, adaptation, distribution and reproduction in any medium or format, as long as you give appropriate credit to the original author(s) and the source, provide a link to the Creative Commons licence, and indicate if changes were made. The images or other third party material in this article are included in the article's Creative Commons licence, unless indicated otherwise in a credit line to the material. If material is not included in the article's Creative Commons licence and your intended use is not permitted by statutory regulation or exceeds the permitted use, you will need to obtain permission directly from the copyright holder. To view a copy of this licence, visit http://creativeco mmons.org/licenses/by/4.0/. The Creative Commons Public Domain Dedication waiver (http://creativecommons.org/publicdomain/ zero/1.0/) applies to the data made available in this article, unless otherwise stated in a credit line to the data. 
pneumoniae, Acinetobacter baumanni, Pseudomonas aeruginosa i Enterobacter species) [4] and recently selected as a high priority pathogen in the global priority pathogens list of the The World Health Organization for the development of new antibiotics [1-3, 5-7].

VRE colonization often precedes infection $[8,9]$ and numerous studies have identified hospital wards as places where VRE colonization poses the greatest risk to human health, in particular hematology, oncology, dialysis, intensive care units (ICUs), geriatrics and acute infectious diseasese wards [2, 3, 10-13]. Previous studies have shown that the prevalence of VRE colonization among hospital patients in Europe varies from 2 to $37 \%$ [3, 14].

Strategies to control the transmission of VRE in hospital settings include screening, early identification of colonization and the isolation of high risk patients $[1,2,5,9,15]$. The information about intestinal carriage and molecular epidemiology of VRE among atrisk patients in the Balkan region is scarce. Therefore, case-based surveillance [16] through which the baseline data can be obtained on the prevalence of VRE intestinal carriage as well as molecular epidemiology studies of circulating VRE stains in high-risk units are needed.

The first case of VRE in Serbia was reported in 2002 in the Clinical Center of Serbia (CCS), the largest tertiary-care teaching hospital in Serbia, which is also a major referal center for neighboring countries of exYugoslavia (Montenegro, Bosna and Herzegovina) [17]. Sixteen years later, according to the data of the Central Asian and European Surveillance of Antimicrobial Resistance network [18], among invasive enterococci in Serbia vancomycin resistance was found in 54\% of Enterococcus faecium and in 5\% of Enterococcus faecalis isolates. These results were suggested to reflect hospital acquired infections and clone dissemination [18].

As hospital acquired VRE infections could be the tip of the iceberg [9] the aim of this study was to provide baseline data on VRE intestinal carriage among patients at high risk departments for VRE colonization in major Serbian university hospitals and to determine the full antibiotic susceptibility profiles along with frequency of resistance to individual drugs, biofilm production capacity and genetic relatedness of the isolated VRE. We also sought to bridge the gaps in current VRE surveillance in the Balkan region regarding screening data for pateints in high risk areas as part of a new concept of case-based surveillance [16] and to enhance the role of microbiology laboratory as a key partner in survaillance of antimicrobial resistance (AMR).

\section{Materials and method}

\section{Study design and setting}

The intestinal VRE reservoir was investigated by multicenter cross-sectional study in six hospital departments of university hospitals in Serbia over the period of 1.5 years (from June 2015 to January 2017): geriatrics, 29 beds; ICUs, 22 beds; hemato-oncology, 25 beds; acute infective disease, 21 beds; hemodialysis, 31 beds.

The sample size was calculated using Epi info ${ }^{\mathrm{TM}} 7$ (CDC, USA) statistical software. Parameters used to calculate the sample size were: expected frequency of colonized patients with VRE strains in the hospital population of $18 \%$ [14], with a $95 \%$ confidence interval and acceptable margin of error of $5 \%$. Accordingly, the sample size was calculated to be 227 .

Participation was voluntary and comprised inpatients aged 18 years and older, of both sexes, who signed informed consent form prior to their inclusion in the study. The study was approved by The Ethical Boards of the included University hospitals: CCS, Zvezdara University Medical Centre (ZvUMC), Zemun University Medical Centre (ZmUMC). Important to note is that Serbia is a middle income Southeastern European country, with about seven million inhabitants, of which roughly $1 / 4$ lives in Belgrade.

\section{Isolation}

Stool samples for VRE testing were collected from 268 inpatients in sterile containers and were processed within $2 \mathrm{~h}$ after collection. Chromogenic agar medium (CHROMID ${ }^{\circledR}$ VRE, bioMerieux, France) was used for VRE screening. Stool samples were directly plated. In accordance with the manufacturers' recommendations, plates were incubated at $36 \pm 1{ }^{\circ} \mathrm{C}$ in ambient air, examined for growth after $24 \mathrm{~h}$ and $48 \mathrm{~h}$ and violet and blue-green colonies were presumptively identified as vancomycin resistant Enterococcus faecium (VREfm) or vancomycin resistant Enterococcus faecalis (VREfs), respectively. Three to four VRE colonies were inoculated into $1 \mathrm{~mL}$ of sterile Tryptic soy broth (TSB, Torlak, Serbia) and overnight cultures were microscopically examined for purity. Overnight cultures were placed in $10 \%$ glycerol and stored at $-72 \pm 1{ }^{\circ} \mathrm{C}$ until future processing. Prior to testing, isolates were subcultured onto Columbia agar with $5 \%$ sheep blood (Torlak, Serbia).

\section{Identification to the species level and antimicrobial susceptibility testing}

Identification and antimicrobial susceptibility testing were performed using the BD Phoenix ${ }^{\mathrm{TM}}$ automated microbiology system (BD, USA) with Gram Positive Combo Panels (PMIC/ID-94, BD, USA). Interpretation of 
results and quality control were performed in accordance with the latest version of the European Committee on Antimicrobial Susceptibility Testing (EUCAST) recommendations (v 10.0) [19].

\section{Molecular identification and detection of resistance and virulence genes}

DNA isolation was performed from the pellet of $5 \mathrm{~mL}$ overnight cultures. The pellet was resuspended in $200 \mu \mathrm{L}$ of TE buffer with $0.1 \mathrm{~mm}$ glass beads. After vortexing egg white lysozyme and achromopeptidase were added and incubated for $2 \mathrm{~h}$ at $36 \pm 1{ }^{\circ} \mathrm{C}$. Two freeze-thaw cycles were performed. Subsequently, $30 \mu \mathrm{l}$ lysis buffer was added and diluted with $180 \mu \mathrm{l}$ buffer $(10 \mathrm{mM}$ Tris- $\mathrm{HCl}$, $\mathrm{pH}$ 8.5). Cell lysates were centrifuged at $16,000 \times g$ for $5 \mathrm{~min}$. DNA from the supernatant was precipitated using ethanol and dissolved in water.

Multiplex polymerase chain reaction (PCR) was performed to detect genes for species identification $\left(d d l_{E}\right.$. faecium,$d d l_{E}$, faccalis $\left._{1}\right)$ and for detection of resistance to vancomycin (van $\mathrm{A}, \operatorname{van} \mathrm{B}, \operatorname{van} \mathrm{C} 1, \operatorname{van} \mathrm{C} 2 / \mathrm{C} 3)$ [20-22]. Another multiplex PCR was performend to detect Quinupristin-dalfopristin $(\mathrm{Q}-\mathrm{D})$ resistance genes $($ vat $\mathrm{D}$, vat $\mathrm{E}$, vgbA, ermB-1) [23].

The presence of six virulence genes was tested in this study: esp, hyl, efaA, asal, gelE, and cpd [24, 25]. In this analysis two multiplex PCRs were performed.

For PCR reactions Fast Gene Taq Ready Mix with dye (NIPPON Genetics, EUROPE GmbH) or Fusion Hot Start II High Fidelity Master Mix (Thermo Scientific, USA) were used as apropriate. Amplified products were analyzed with $1.5 \% \mathrm{w} / \mathrm{v}$ agarose gel. As molecular weight size marker 100 bp DNA Ladder (H3 RTU, NIPPON Genetics, EUROPE GmbH) was used.

\section{MLVA typing}

VRE $f m$ isolates were genotyped using Multiple-locus variable-number tandem-repeat (VNTR) analysis (MLVA) which was performed using a previously described method [26] with minor modification. An initial denaturation at $95{ }^{\circ} \mathrm{C}$ for 5 min was used for all cycles. VNTR-2 Touch Down (TD) PCR was done with decreasing annealing temperature for $0.7^{\circ} \mathrm{C}$ at each cycle during the next 9 cycles and during the next 35 cycles, an annealing temperature of $63^{\circ} \mathrm{C}$ was used. VNTR-9 TD PCR was done as follows: 10 cycles of $30 \mathrm{~s}$ at $94{ }^{\circ} \mathrm{C}, 30 \mathrm{~s}$ at $70{ }^{\circ} \mathrm{C}$ and $1 \mathrm{~min}$ at $72{ }^{\circ} \mathrm{C}$ with annealing temperature decreasing for $0.6{ }^{\circ} \mathrm{C}$ at each cycle during the next 9 cycles and during the next 35 cycles an annealing temperature of $64{ }^{\circ} \mathrm{C}$ was used. For VNTR-7, VNTR-8 and VNTR-10 PCR involving 35 cycles and an annealing temperature of $55^{\circ} \mathrm{C}$ was used.
Cluster analysis of MLVA data was performed by BioNumerics software (version 7; Applied Maths) using UPGMA (Unweighted-pair group) clustering method with categorical coefficient of similarity. Cut-off value for clustering was set on $85 \%$ of similarity with a minimum of 2 members in a cluster and Simpson's index of diversity was calculated. MLVA types (MTs) were assigned by comparison with the existing MLVA base [26]. For new combinations of MLVA profiles, new MTs were assigned. They were named according to the country of isolation (SRB) followed by incrising number obtained from phylogenetic analyses.

\section{Biofilm formation assay}

The ability of isolated VRE to form biofilm was tested by microtiter plate method [27] in 96-well flat-bottomed polystyrene plates using TSB supplemented with 1\% glucose and crystal violet staining. The absorbance of each plate was measured at $580 \mathrm{~nm}$ using a microtiter plate reader and the results were calculated according to Stepanović et al. [27].

\section{Results}

A total of 268 inpatients were tested, of which 77 (28.7\%) were intestinal carriers of VREfm strains, with $v a n A$ genotypic profile and VanA phenotypic profile. The frequencies of VRE $f m$ carriage among patients hospitalized in the aforementioned departments are shown in Table 1.

The analysis of antibiotic susceptibility, shown in Table 2, exposed high prevalence of multidrug-resistant VRE strains. In addition to vancomycin and teicoplanin resistance, 23.4\% (18/77) VREfm isolates were resistant to all remaining antimicrobial drugs, except linezolid and tigecyclin to which all the isolates were susceptible. Additionaly, 38.9\% of VRE $f m$ strains were resistant to Q-D.

Phenotypic resistance profiles of the isolated VRE $f m$ strains are shown in Table 3.

Table 1 Distribution of isolated Vancomycin-resistant Enterococcus faecium (VREfm) stratified by investigated hospital departments

\begin{tabular}{lll}
\hline Department & \multicolumn{2}{l}{ VREfm } \\
\cline { 2 - 3 } & $\mathrm{n} / \mathrm{N}$ & $\%$ \\
\hline Geriatrics & $23 / 54$ & 42.6 \\
ICUs & $16 / 40$ & 40.0 \\
Hemato-Oncology & $22 / 79$ & 27.9 \\
Acute infectious diseases & $10 / 44$ & 22.7 \\
Hemodialysis & $6 / 51$ & 11.7 \\
Total & $77 / 268$ & 28.7 \\
\hline
\end{tabular}

ICUs intensive care units, $n$ number of VRE positive patients, $N$ number of participants 
Table 2 Antibiotic resistant profile of Vancomycin-resistant Enterococcus faecium (VREfm) isolates $(\mathrm{N}=77)$

\begin{tabular}{lll}
\hline Antimicrobial drug & \multicolumn{2}{l}{ VREfm isolates } \\
\cline { 2 - 3 } & Resistant & \\
\cline { 2 - 3 } & $n$ & 92.2 \\
AMP & 71 & 92.2 \\
IMP & 71 & 97.4 \\
CIP & 75 & 97.4 \\
LEV & 75 & 89.6 \\
GEN-HLS & 69 & 94.8 \\
STR-HLS & 73 & 100 \\
TEI & 77 & 38.9 \\
Q-D & 30 & 0 \\
TIG & 0 & 0 \\
LIN & 0 & \\
\hline
\end{tabular}

$n$ number of VREfm isolates resistant to tested antimicrobial drug, AMP ampicillin, IMP imipenem, CIP ciprofloxacin, LEV levofloxacin, GEN-HLS gentamicin-high level aminoglycoside resistance, STR-HLS streperomicin-high level aminoglycoside resistance, $T E I$ teicoplanin, $Q-D$ quinupristin-dalfopristin, TIG tigecyclin, LIN linezolid

Table 3 Phenotipic profile of isolated Vancomycin-resistant Enterococcus faecium (VREfm)-antimicrobial susceptibility pattern

\begin{tabular}{lll}
\hline Antimicrobial susceptibility pattern & \multicolumn{2}{l}{ Resistant } \\
\cline { 2 - 3 } & $\mathrm{n}$ & $\%$ \\
\hline AMP IMP CIP-LEVO GEN-HLS STR-HLS TEI & 48 & 62.3 \\
AMP IMP CIP-LEVO GEN-HLS STR-HLS TEI Q-D & 18 & 23.4 \\
CIP-LEVO GEN-HLS STR-HLS TEI Q-D & 4 & 5.2 \\
AMP IMP CIP-LEVO STR-HLS TEI Q-D & 2 & 2.6 \\
AMP IMP CIP-LEVO GEN-HLS TEI Q-D & 2 & 2.6 \\
CIP-LEVO GEN-HLS TEI & 1 & 1.3 \\
AMP IMP TEI Q-D & 1 & 1.3 \\
TEI Q-D & 1 & 1.3 \\
Total & 77 & 100 \\
\hline
\end{tabular}

AMP ampicillin, IMP imipenem, CIP ciprofloxacin, LEV levofloxacin, GEN-HLS gentamicin-high level aminoglycoside resistance, STR-HLS streperomicin-high level aminoglycoside resistance, $T E I$ teicoplanin, $Q-D$ Quinupristin-dalfopristin

Biofilm quantitative test showed that $20.7 \%(16 / 77)$ bacterial strains were biofilm producers with different capacity. Biofilm production capacities of given pathogens are shown in Table 4.

Molecular genetic analysis for molecular confirmation of Q-D resistance was performed on DNA samples obtained from purified isolates. The analysis confirmed that all isolates showing phenotypic resistance carried the ermB-1 gene. Other genes associated with resistance to $\mathrm{Q}-\mathrm{D}(v a t \mathrm{D}, v a t \mathrm{E}, v g b \mathrm{~A})$ were not detected. Genetic analysis of the presence of virulence genes revealed that
Table 4 Phenotipic profile of isolated vancomycin-resistant Enterococcus faecium (VREfm) — biofilm production capacity

\begin{tabular}{lll}
\hline Biofilm production capacity & N & \\
\hline Yes & 16 & 20.8 \\
Weak & 9 & 11.7 \\
Moderate & 4 & 5.2 \\
Strong & 3 & 3.9 \\
No & 61 & 79.2 \\
Total & 77 & 100 \\
\hline
\end{tabular}

weak biofilm producer-category $1,+;$ moderate biofilm producer-category 2 , $++;$ strong biofilm producer-category $3,+++;$ no-no biofilm formation

Table 5 Characteristics of isolated Vancomycin-resistant Enterococcus faecium (VREfm) - genotypic profile

\begin{tabular}{|c|c|c|}
\hline Genotypic profile & $\mathrm{N}$ & $\%$ \\
\hline \multicolumn{3}{|l|}{ Identification genes } \\
\hline$\left.d d\right|_{E \text {.faecium }}$ & 77 & 100 \\
\hline$\left.d d\right|_{E_{\text {ffaecalis }}}$ & 0 & 0 \\
\hline \multicolumn{3}{|c|}{ Vancomycin resistance genes } \\
\hline $\operatorname{vanA}$ & 77 & 100 \\
\hline $\operatorname{van} B$ & 0 & 0 \\
\hline $\operatorname{vanC}(\mathrm{C} 1$ and $\mathrm{C} 2 / 3)$ & 0 & 0 \\
\hline \multicolumn{3}{|l|}{ Q-D resistance genes } \\
\hline vatD & 0 & 0 \\
\hline vatE & 0 & 0 \\
\hline$v g b A$ & 0 & 0 \\
\hline ermB-1 & 30 & 38.9 \\
\hline \multicolumn{3}{|l|}{ Virulence genes } \\
\hline esp & 65 & 84 \\
\hline$e f a A$ & 55 & 71.2 \\
\hline hyl & 42 & 54.5 \\
\hline gelE & 9 & 11.6 \\
\hline asal & 18 & 23.4 \\
\hline$c p d$ & 9 & 11.6 \\
\hline \multicolumn{3}{|c|}{ Number of virulance genes } \\
\hline 0 & 8 & 10.4 \\
\hline 1 & 4 & 5.2 \\
\hline 2 & 20 & 26.0 \\
\hline 3 & 34 & 44.0 \\
\hline 4 & 4 & 5.2 \\
\hline 5 & 4 & 5.2 \\
\hline 6 & 3 & 4.0 \\
\hline
\end{tabular}

$\left.d d\right|_{E \text {.faecium, }} \mathrm{D}$-alanine-D-alanine ligase gene specific for $E$. faecium; $\left.d d\right|_{E \text {.faecalist }}$ $D$-alanine-D-alanine ligase gene specific for $E$. faecalis; van $A$, type $A$ vancomycin resistance; van B, type B vancomycin resistance; vanC (C1 and C2/3) — type C vancomycin resistance; $v a t(D)$ and $\operatorname{vat}(\mathrm{E})$, streptogramin $A$ resistance; $v g b(\mathrm{~A})$ and ermB-1- streptogramin B resistance; esp, Enterococcal surface protein; hyl, hyaluronidase; efaA, cell wall adhesine; asa1, aggregation substance; $g$ elE, gelatinase; $c p d$, sex pheromones 


\section{(See figure on next page.)}

Fig. 1 Dendogram of Vancomycin-resistant Enterococcus faecium isolates using Unweighted pair group clustering method. VNTR, variable-number tandem-repeat; MT-MLVA type; HO, Haematology-Oncology; Ger, Geriatrics; ICU, Intensive Care Units; AID, Acute Infection Diseases; HD, Haemodyalisis; AMP, Ampicillin; IMP, Imipenem; CIP, Ciprofloxacin; LEV, Levofloxacin; GEN-HLS, Gentamicin-high level aminoglycoside resistance; STR-HLS, Streperomicin-high level aminoglycoside resistance; VAN, Vancomycin; TEl, Teicoplanin; Q-D, Quinupristin-dalfopristin; ddl E.faecium D-alanine-D-alanine ligase gene specific for $E$. faecium; $\left.d d\right|_{E \text {.faecalis' }}$ D-alanine-D-alanine ligase gene specific for E. faecalis; van $A$, type $A$ vancomycin resistance; van $B$, type $B$ vancomycin resistance; vanC (Cland C2/3) - type $C$ vancomycin resistance; vat(D) and vat $(\mathrm{E})$, streptogramin $A$ resistance; $v g b(A)$ and ermB-1, streptogramin B resistance; esp, Enterococcal surface protein; hyl, hyaluronidase; efaA, cell wall adhesine; asal, aggregation substance; gelE, gelatinase; $c p d$, sex pheromones; $\mathrm{N}$, no virulence genes; + , weak biofilm producer; ++ , moderate biofilm producer; +++ , strong biofilm producer;-, no biofilm production

84\% VRE $f m$ isolates harbored the esp gene, while three isolates harbored all tested virulence genes (Table 5).

In order to assess the genetic relatedness of the VRE $f m$ isolates a standard MLVA analysis was performed on isolated DNA samples. A total of 72/77 (93.5\%) isolates generated a MLVA profile, while MLVA profiles could not be assigned for 5/77 (6.5\%) isolates. MLVA revealed 29 different MTs, of which 25 were not previously detected and had unique profiles. Simpson's index of diversity was 94\%.

Genetic relatedness analysis of the isolates revealed 13 clusters which comprised 56/72 (77.7\%) of the isolated VRE $f m$ strains (Fig. 1). Three of 13 clusters included 12 (SRB2), 9 (SRB16) and 7 (MT161) isolates each, while the 10 remaining clusters included 2 to 4 isolates. The remaining 16/72 (22.2\%) VRE $f m$ isolates had unique genotypes and were not clonally related to the other isolates. Next we sought to determine the association of genotype with the location of inpatient from which the VRE $f m$ was isolated. We detected that the isolates belonging to the larger clusters were dispersed among different hospital departments (Fig. 1). Interestingly, isolates belonging to the 3 minor clusters (SRB6, SRB9, SRB12) originated from hematology department only.

\section{Discussion}

In the last 15 years, VREs derived as a major cause of HA infections worldwilde due to intrinsic and acquired resistance to various classes of antimicrobial drugs, scarcity in treatment options and high tendency to become endemic in the hospital environment [28]. European Centre for Disease Prevention and Control points out an increasing trend in the number of VRE $f m$ infections and VRE $f m$ associated deaths in European countries and its contribution to European health burden of antimicrobial resistance, highlighting the urgency for better understanding molecular epidemiology of circulating VRE [28]. VRE colonization often precedes infection and selective screening of at-risk patients for VRE colonization is one of the infection controling measures recommended for prompt identification and isolation of carriers, thus being the crutial step in control of patient-to-patients transmission $[2,3,8,9]$.

This study presents the first case-based surveillance report on the prevalence of VRE intestinal carriage among high-risk inpatients in Serbia and partially in the ex-Yugoslav region. The intention was to broaden awareness and increase knowledge on VRE intestinal carriage in the Balkan region as well as to provide baseline data regarding molecular epidemiology and genetic diversity of circulating VRE strains in Serbia.

VRE carriage was found in $28.7 \%$ of the study population. This frequency is high compared to low prevalence European countries, e.g. the Netherlands (2\%) and Belgium (3.5\%) but similar to frequencies reported in studies from Ireland (19.1\%), United States of America (USA) (33\%) and certain regions of France (37\%) [3, 14, 29]. However, comparison of the data form different countries is very difficult and should be done carefully as there are many variables to be taken into account. For example, there could be differences in the study period, the primary diagnosis, in the particular hospital units investigated, in the type of the analyzed specimens and in the media used for isolation. Additionally there could be differences in reporting of acquired and intrinsic resistance profiles which may have significant impact on the obtained results.

In this study, we used the direct plating method of stool specimens onto selective chromogenic agar medium for the detection of the acquired VRE $f s$ and VRE $f m$ strains. Several studies $[3,30]$ evaluated usefulness of the selective chromogenic media in the detection of VRE colonization, which can explain the high rate of recovery of VRE in our study.

All detected VRE strains were VRE $f m$ with vanA genotypic profile and VanA phenotypic profile, which is typical for European counties [1,2]. Similar to the study of Whelton et al. [29], neither VREfs nor Enterococcus gallinarum/casseliflavus were isolated in our research. Predominance of multidrug-resistant VRE $f m$ may indicate that the strategy of making hospital environment its novel ecological niche has been successfully mastered by the bacteria. This is in line with previous 


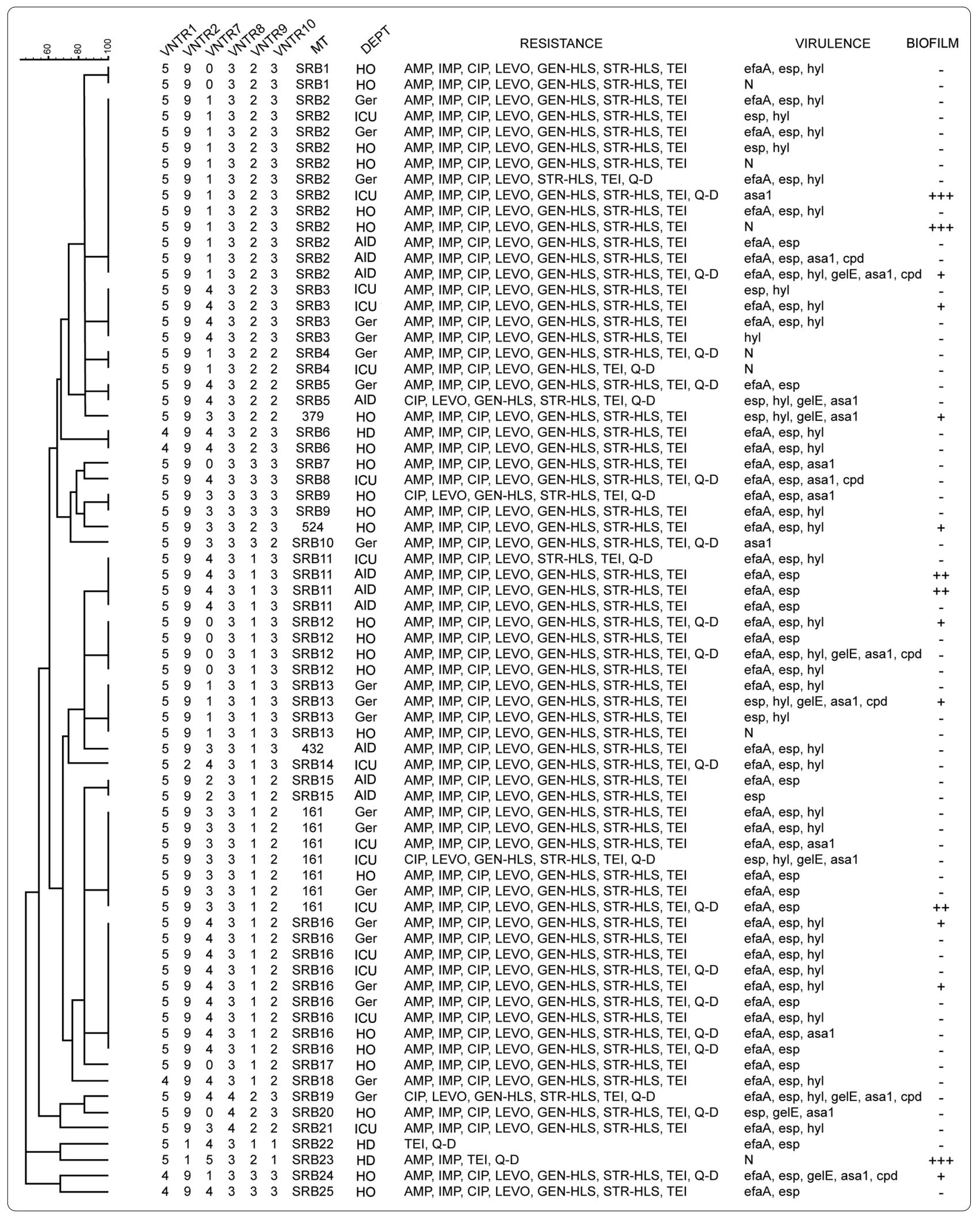


conclusions that multidrug resistant $E$. faecium is a dominant reservoir of acquired vancomycin resistance in hospitals, particularly among immunocompromised patients, resulting in dissemination of resistance genes among the bacterial population upon selective antibiotic pressure $[2,5]$.

Interestingly, high rate of resistance to Q-D (38.9\%) in VRE $f m$ isolates was found. Q-D is a streptogramin, antimicrobial drug licensed for treatment of infections caused by MDR bacteria, including VRE $f m$. Although resistance to this drug among VRE $f m$ is known, it is rare in human isolates [31]. Moreover, Q-D is not licensed for clinical use is Serbia. Thus, the high frequency of resistance to Q-D among VRE $f m$ strains in Serbia is of special concern for two reasons. Firstly, the ability of VRE $f m$ strains resistant to Q-D to spread and cause healthcareassociated outbreaks is potentially high. Secondly, treatment options for infections caused by these strains are narrowed. We found that all VRE $f m$ isolates resistant to Q-D harbored the ermB-1 gene, that conferred resistance to macrolides, lincozamine and streptogramine B, which is in line with previous findings [31]. Considering that $\mathrm{Q}-\mathrm{D}$ is not licensed for clinical use in Serbia and that it has never been a part of therapeutic protocols in Serbia, possible explanation for Q-D resistance in addition to vancomycin resistance is the exchange of resistance genes between E. faecium from human and animal sources, as resistance to $\mathrm{Q}-\mathrm{D}$ is common in isolates retrieved from animals and is linked to the use of virginamycin as growth promotion factor [23, 31].

In order to better understand VRE colonization and its implication in infection, we studied virulence factors. Obtained results showed that almost all tested virulence genes (esp, hyl, efa $\mathrm{A}, a s a 1, g e l \mathrm{E}, c p d)$ were present in analysed VRE $f m$ strains. Additionally, $58.4 \%$ of the isolates were found to carry a minimum of 3 known virulance genes, which is in contrast to the findings of Billström [32] where only $2 \%$ of isolates carried multiple virulence genes. Our data showed that VRE $f m$ with all the virulence factors seems to be equipped to survive and spread in hospital environment. This ability to collect various virulence genes may be explained by adaptive mechanisms, e.g. "genetic capitalism" due to the gene transfer and recombination [33-35]. Recently, Freitas et al. [36] has shown that the number of virulence genes present in VRE $f m$ correlate well with ampicillin-resistant phenotype and that VRE $f m$ strains with higher ampicillin minimum inhibitory concentration values have higher number of virulence genes, which contributes to their pathogenicity. Almost all VREfm strains from our study were resistant to ampicillin and most of them contained several virulence genes and therefore might pose a serious risk for infection in at-risk patients.
Finaly, in our study we performed MLVA typing, fast, cheap, easy-to-use, PCR-based method recommended as an initial rapid screening typing tool for the analysis of phylogenetic relatedness of isolated VRE $f m$ in hospital settings [26, 37]. The largest isolated clusters (SRB2, MT 161 and SRB16) represent single-locus variant (SLV) or double locus variants of MT- 340 and MT-159, known to cause infections in hospitalizied patients in Serbia [38, 39]. Namely, MLVA-C1 genogroup comprises the majority of epidemic and clinical VRE $f m$ isolates, with MT-1 and its SLV MT-159, being the most common types associated with hospital outbreaks and invasive infections in the last decade in Europe [39]. Therefore, our results might indicate an evolution in hospital-adopted clones which might happen sporadically. Simpson's index of diversity demonstrated high diversity among the isolates, implying phylogenetic unrelatedness. Being that we found five isolates which could not be classified by MLVA, possibly as a result of lacking one to three VNTR loci, as well as 25 unique MTs, one could speculate that the origin of vancomycin resistance in E. faecium in our study is due to the horizontal gene tranfer and selective antibiotic pressure [33, 34, 40]. Indeed, high prevalence of VRE $f m$ was found in geriatrics departments where vancomycin is a common agent for Clostridioides difficile infection treatment. Also, broad-spectrum antimicrobial therapy is the most common therapeutic protocol in ICUs, while vancomycin is commonly used as part of empiric therapy of febrile neutropenia in hematological patients [10, 13]. Hence, unlike the rest of Europe, where the origin of VRE in hospitals is connected to the usage of avoparcin in farm animals, in Serbia we noticed a different scenario, likely similar to that in the USA, where VRE in hospitals is due to the overuse of vancomycin [2].

Although definitive proof of patient-to-patient transmission requires high resolution typing techniques like multilocus sequence typing and whole genome sequencing $[9,41]$, we consider MLVA to be a reliable typing method in the context of infection control surveillance where primary focus is the exclusion of clonal relatedness among isolates and timely alert if an outbreak investigation is about to commence.

\section{Conclusion}

The obtained prevalence of VRE intestinal carriage among high-risk inpatients in Serbia is higher than the European average, with high percentage of multidrugresistance and the ability of biofilm production. Various virulence genes might affect the pathogenicity of the strains. Reporting full antimicrobial resistance profiles along with the frequency of resistance to individual drugs for at-risk population is a change in approch to reporting AMR data and a step closer to the new 
concept of case-based surveillance of AMR. Of particular concern is the emergence of resistance to Q-D that has never been licensed for clinical use in Serbia nor has ever been a part of therapeutic protocols in our country. The illicit usage of antibiotics in animal farming could be implicated. MLVA revealed polyclonal setting with 25 unique MT profiles that were most likely selected through antibiotic pressure. This study also contributes to the investigation of VRE $f m$ genotype distribution in the neighboring countries within the Balkan peninsula. Close monitoring of the pattern of resistance, implementation of specific guidelines, cleaning procedures and antibiotic stewardship policy, as well as the introduction of VRE screening among at-risk inpatients, as part of active surveillance are urgently needed in Serbia.

\section{Abbreviations}

AMR: Antimicrobial resistance; CCS: Clinical Center of Serbia; EUCAST: European Committee on Antimicrobial Susceptibility Testing; HA: Health-care associated; ICUs: Intensive care units; MLVA: Multiple-locus variable-number tandem-repeat analysis; MTs: MLVA types; PCR: Polymerase chain reaction; Q-D: Quinupristin-dalfopristin; SLV: Single-locus varian; SRB: Serbia; TE buffer: Tris EDTA buffer; TSB: Tryptic soy broth; TD PCR: Touch down PCR; UPGMA: Unweighted-pair group; USA: Unites States of America; VNTR: Variable-number tandem-repeats; VRE: Vancomycin resistant enterococci; VREfm: Vancomycin resistant Enterococcus faecium; VREfs: Vancomycin resistant Enterococcus faecalis; ZvUMC: Zvezdara University Medical Centre; ZmUMC: Zemun University Medical Centre.

\section{Acknowledgements}

This investigation was supported by the Ministry of Education, Science and Technological Development of the Republic of Serbia (Grant Nos. 175039 and No.175046)

\section{Authors' contributions}

Conceptualization: IC, LjMD, AG, AJ; performed the experiments: AJ, RM; analyzed the data: IC, AJ, LJMD; writing- original draft: AJ; writing—-review and editing: IC, LjMD, RM. All authors read and approved the final manuscript.

\section{Funding}

The authors have no funding to report.

\section{Availability of data and materials}

All data generated or analyzed during this study are included in this published article.

\section{Declarations}

\section{Ethics approval and consent to participate}

The study was approved by The Ethical Boards of the included university hospitals in Belgrade, Serbia: (Reference number of Ethical Boards: CCS, no: 05/2016; ZvUMC, no: 05/2015; ZmUMC, no: 02/2015). Informed consent was obtained from all individual participants included in the study. All procedures performed in studies involving human participants were in accordance with the ethical standards of the institutional and/or national research committee and with the 1964 Helsinki Declaration and its later amendments or comparable ethical standards.

\section{Consent for publication}

Not applicable.

\section{Competing interests}

The authors declare that they have no competing interests.

\section{Author details}

1 Department of Bacteriology, Institute of Virology, Vaccines and Sera "Torlak", Vojvode Stepe 458, 11152 Belgrade, Serbia. ${ }^{2}$ Department of Epidemiology, Institute of Epidemiology, Faculty of Medicine, University of Belgrade, Visegradska 26a, PO Box 20, 11129 Belgrade, Serbia. ${ }^{3}$ Department of Bacteriology, Institute of Microbiology and Immunology, Faculty of Medicine, University of Belgrade, Dr Subotića 1, 11000 Belgrade, Serbia.

Received: 17 February 2021 Accepted: 9 March 2021

Published online: 20 March 2021

\section{References}

1. Werner G, Coque TM, Hammerum AM, Hope R, Hryniewicz W, Johnson $A$, et al. Emergence and spread of vancomycin resistance among enterococci in Europe. Euro Surveill. 2008;13(47):1-11.

2. Bonten MJ, Willems R, Weinstein RA. Vancomycin-resistant enterococci: why are they here, and where do they come from? Lancet Infect Dis. 2001;1(5):314-25.

3. Faron ML, Ledeboer NA, Buchan BW. Resistance mechanisms, epidemiology, and approaches to screening for vancomycin-resistant Enterococcus in the health care setting. J Clin Microbiol. 2016;54(10):2436-47.

4. Rice LB. Federal funding for the study of antimicrobial resistance in nosocomial pathogens: no ESKAPE. J Infect Dis. 2008;197(8):1079-81.

5. Mutters NT, Mersch-Sundermann V, Mutters R, Brandt C, Schneider-Brachert W, Frank U. Control of the spread of vancomycin-resistant enterococci in hospitals: epidemiology and clinical relevance. Dtsch Arztebl Int. 2013;110(43):725-32.

6. Tacconelli E, Carrara E, Savoldi A, Harbarth S, Mendelson M, Monnet DL, et al. Discovery, research, and development of new antibiotics: the WHO priority list of antibiotic-resistant bacteria and tuberculosis. Lancet Infect Dis. 2018;18(3):318-27.

7. Tacconelli E, Margini. Global priority list of antibiotic-resistant bacteria to guide research, discovery, and development of new antibiotics. Geneva: World Health Organization; 2017. http://www.cdc.gov/drugresistance/ threat-report-2013/. Accessed 27 July 2020.

8. Zirakzadeh A, Patel R. Vancomycin-resistant enterococci: colonization, infection, detection, and treatment. Mayo Clin Proc. 2006;81 (4):529-36.

9. Zhou X, Willems RJL, Friedrich AW, Rossen JWA, Bathoorn E. Enterococcus faecium: from microbiological insights to practical recommendations for infection control and diagnostics. Antimicrob Resist Infect Control. 2020;9(1):1-13.

10. Pan S, Wang J, Chen Y, Chang Y, Chen M, Chang C. Incidence of and risk factors for infection or colonization of vancomycin-resistant enterococci in patients in the intensive care unit acute renal failure. PLOS ONE. 2012;7(10):1-8

11. Shaghaghian S, Pourabbas B, Alborzi A, Askarian M, Mardaneh J. Vancomycin-Resistant Entrococci colonization in chronic hemodialysis patients and its risk factors in southern. Iran Red Crescent Med J. 2012;14(3):686-91.

12. Gudiol C, Ayats J, Camoez M, Domínguez MÁ, García- C, Bodro M, et al. Increase in bloodstream infection due to vancomycin- susceptible enterococcus faecium in cancer patients : risk factors, Molecular Epidemiology and Outcomes. PLoS ONE. 2013;8(9):1-8.

13. Deplano A, Denis O, Nonhoff C, Rost F, Byl B, Jacobs F, et al. Outbreak of hospital-adapted clonal complex-17 vancomycin-resistant Enterococcus faecium strain in a haematology unit: Role of rapid typing for early control. J Antimicrob Chemother. 2007;60(4):849-54.

14. Gambarotto K, Ploy MC, Turlure P, Grélaud C, Martin C, Bordessoule D, et al. Prevalence of vancomycin-resistant enterococci in fecal samples from hospitalized patients and nonhospitalized controls in a cattlerearing area of France. J Clin Microbiol. 2000;38(2):620-4.

15. Faron ML, Ledeboer NA, Buchan BW. Resistance mechanisms, epidemiology, and approaches to screening. J Clin Microbiol. 2016;54(10):2436-47.

16. Ryu S, Cowling BJ, Wu P, Olesen S, Fraser C, Sun DS, et al. Case-based surveillance of antimicrobial resistance with full susceptibility profiles. JAC-Antimicrobial Resist. 2019;1(3):1-5. 
17. Stosovic B, Stepanovic S, Donabedian S, Tosic TJM. Vancomycin- resistant Enterococcus faecalis in Serbia. Emerg Infect Dis. 2004;10(1):157-8.

18. WHO/Europe / Central Asian and European Surveillance of Antimicrobia Resistance (CAESAR). http://www.euro.who.int/en/health-topics/disea se-prevention/antimicrobial-resistance/surveillance/central-asian-andeuropean-surveillance-of-antimicrobial-resistance-caesar. Accessed 7 June 2020

19. The European Committee on Antimicrobial Susceptibility Testing. http:// www.eucast.org/. Accessed 21 Feb 2020

20. Dutka-malen S, Evers S, Courvalin P. Detection of glycopeptide resistance genotypes and identification to the species level of clinically relevant Enterococci by PCR. J Clin Microbiol. 1995;33(1):24-7.

21. Dutka-Malen S, Evers S, Courvalin P. Detection of glycopeptide resistance genotypes and identification to the species level of clinically relevant enterococci by PCR. ERRATUM J Clin Microbiol. 1995;33(5):1434.

22. Poulsen RL, Pallesen LV, Frimodt-Møller N, Espersen F. Detection of clinical vancomycin-resistant enterococci in Denmark by multiplex PCR and sandwich hybridization. APMIS. 1999;107(1-6):404-12.

23. Soltani M, Beighton D, Philpott-Howard J, Woodford N. Mechanisms of resistance to quinupristin-dalfopristin among isolates of Enterococcus faecium from animals, raw meat, and hospital patients in western Europe. Antimicrob Agents Chemother. 2000;44(2):433-6.

24. Vankerckhoven V, Van AT, Vael C, Lammens C, Chapelle S, Rossi R, et al. Development of a multiplex PCR for the detection of asa1, gelE, cylA, esp, and hyl GENES in Enterococci and survey for virulence determinants among european hospital isolates of Enterococcus faecium. J Clin Microbiol. 2004;42(10):4473-9.

25. Eaton TJ, Gasson MJ. Molecular screening of Enterococcus virulence determinants and potential for genetic exchange between food and medical isolates. Appl Environ Microbiol. 2001;67(4):1628-35.

26. Top J. MLVA-typing - UMC Utrecht. https://www.umcutrecht.nl/en/Resea rch/Miscellaneous/MLVA-typing. Accessed 23 Nov 2019.

27. Stepanović S, Vuković D, Hola V, Di BG, Djukić S, Ćircović l, et al. Quantification of biofilm in microtiter plates. APMIS. 2007;115(8):891-9.

28. European Centre for Disease Prevention and Control. Surveillance of antimicrobial resistance in Europe 2018. Stockholm: ECDC; 2019.

29. Whelton E, Lynch C, Reilly BO, Corcoran D, Cryan B, Keane SM, et al. Vancomycin-resistant enterococci carriage in an acute Irish hospital. J Hosp Infect. 2016;93(2):175-80. https://doi.org/10.1016/j.jhin.2016.03.005.

30. Kallstrom G, Doern CD, Michael W, Kallstrom G, Doern CD, Dunne WM Evaluation of a chromogenic agar under development to screen for VRE colonization. J Clin Microbiol. 2010;48(3):999-1001.
31. Donabedian SM, Perri MB, Vager D, Hershberger E, Malani P, Simjee S, et al Quinupristin-dalfopristin resistance in Enterococcus faecium isolates from humans, farm animals, and grocery store meat in the United States. J Clin Microbiol. 2006;44(9):3361-5.

32. Billström H, Lund B, Sullivan $\AA$, Erik C. Virulence and antimicrobial resistance in clinical Enterococcus faecium. Int J Antimicrob Agents. 2005:2008(32):374-7

33. Leavis HL, Bonten MJM, Willems RJL. Identification of high-risk enterococcal clonal complexes : global dispersion and antibiotic resistance. Curr Opin Microbiol. 2006;9:454-60.

34. Baquero F, Martinez JL. Ecology and evolution of antibiotic resistance. Environ Microbiol Rep. 2009;1:469-76.

35. Baquero F. From pieces to patterns : evolutionary engineering in bacterial pathogens. Nat Rev Microbiol. 2004;2(June):510-8.

36. Freitas AR, Tedim AP, Novais C, Coque TM, Peixe L. Distribution of putative virulence markers in Enterococcus faecium: towards a safety profile review. J Antimicrob Chemother. 2018;73(2):306-19.

37. Top J, Schouls LM, Bonten MJM, Willems RJL. Multiple-locus variablenumber tandem repeat analysis, a novel typing scheme to study the genetic relatedness and epidemiology of Enterococcus faecium isolates. J Clin Microbiol. 2004;42(10):4503-11.

38. Jovanović M, Top J, Majoor E, Zervos M, Tošić T, Stošović B, et al. Multiplelocus variable number tandem repeat analysis typing of vancomycinresistant Enterococcus faecium in Serbia. Infect Control Hosp Epidemiol. 2014;34(12):12-5.

39. Billström H, Top J, Edlund C, Lund B. Frequent occurrence of multidrugresistant CC17 Enterococcus faecium among clinical isolates in Sweden. J Appl Microbiol. 2010;108(5):1810-6.

40. Hegstad K, Mikalsen T, Coque TM, Werner G, Sundsfjord A. Mobile genetic elements and their contribution to the emergence of antimicrobial resistant Enterococcus faecalis and Enterococcus faecium. Clin Microbiol Infect. 2010;16:541-54.

41. Van SW, Willems RJL. Genome-based insights into the evolution of enterococci. Clin Microbiol Infect. 2010;16:527-32.

\section{Publisher's Note}

Springer Nature remains neutral with regard to jurisdictional claims in published maps and institutional affiliations.
Ready to submit your research? Choose BMC and benefit from:

- fast, convenient online submission

- thorough peer review by experienced researchers in your field

- rapid publication on acceptance

- support for research data, including large and complex data types

- gold Open Access which fosters wider collaboration and increased citations

- maximum visibility for your research: over $100 \mathrm{M}$ website views per year

At BMC, research is always in progress.

Learn more biomedcentral.com/submissions 